\title{
Diffractive elements for correction of chromatic aberrations of illumination systems
}

\author{
Marek Škereň ${ }^{1, a}$, Jakub Svoboda ${ }^{1}$, Milan Květoň ${ }^{1}$, Jaroslav Hopp ${ }^{1}$, Martin Possolt ${ }^{1}$, and Pavel Fiala ${ }^{1}$ \\ 1 Czech Technical University in Prague, Faculty of Nuclear Sciences and Physical Engineering, Břehová 7, 115 19 Prague, \\ Czech Republic
}

\begin{abstract}
In this paper, the application of synthetic diffractive elements in white light illumination systems is presented. The elements are used for correction of chromatic aberration. The fabrication technology is also presented, which is compatible wit the commonly used molding process for fabrication of plastic optical elements.
\end{abstract}

\section{Introduction}

In comparison with the conventional (refractive) optical systems, the diffractive elements can offer higher flexibility for some applications. Today, the diffractive optical components can be found in many applications from simple illumination systems to hybrid lenses in some photographic objectives. The attractivity of the diffractive elements for the producers is in the fact that the mass, dimensions, and replication costs of the optical system can be significantly reduced. The diffractive elements can be used both separately or as a part of a hybrid refractive-diffractive system. The diffractive elements usually bring also specific problems which are directly connected with basic characteristics of the diffraction process. The typical drawback of a diffractive element is its strong dispersive behavior. However, since the dispersion of the diffractive structure is opposite to that of common refractive element, the combination of such elements can lead to a dispersion-free system [1-3].

In this paper, an application of the diffractive element for compensation of the chromatic aberration of specific illumination system is presented. The illumination system consists of a light source and a single optical element, which images the light source to the desired plane (figure 1).

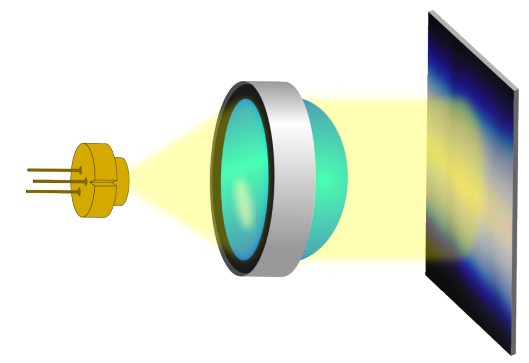

Figure 1. The illumination system with significant chromatic aberration.

Because of complicated aspheric shape of the imaging lens and strong dispersion of its material, the system suffers

\footnotetext{
${ }^{a}$ e-mail: marek.skeren@fjfi.cvut.cz
}

from significant chromatic aberration (the lens is molded from a plastic material like polycarbonate or similar). Since the illumination system is supposed to be used in headlights of an automobile, the compensation of the chromatic aberration is crucial. To compensate for this defect, a correction element has been proposed, which can be advantageously realized in a form of the diffractive structure. If the diffractive element is directly attached to the main imaging lens, the whole imaging system can be manufactured in one step during the molding process. The correction element was designed in ZEMAX software and fabricated using an in-house developed matrix laser writer and injection molding technology. To fabricate such an element for reasonable costs, suitable technology capable of creating large area aperiodic micro-structures with high resolution must be used.

\section{Design of the elements}

The diffractive element was designed to correct the chromatic aberration of a particular sample of aspheric lens. The lens was made from a polycarbonate using the injection molding technology (index of refraction of polycarbonate is $n \sim 1.6$ and Abbe number $V_{d}=27.56$ ). To reduce the costs of the manufacturing process, the profile of the diffractive element should be transferred to the mold tool and the whole assembly of the lens and the compensating element will be manufactured at once.

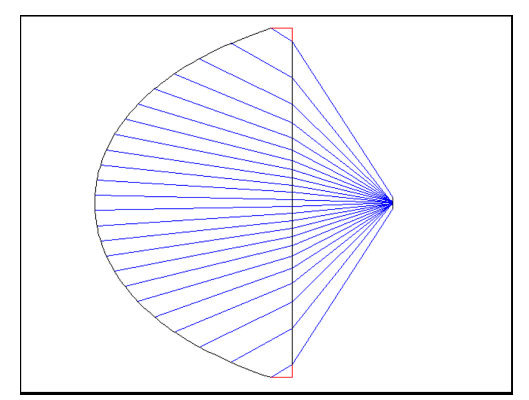

Figure 2. Design layout. The correction element is attached directly to the planar surface of the aspheric lens.

This is an Open Access article distributed under the terms of the Creative Commons Attribution License 2.0, which permits unrestricted use, distribution, and reproduction in any medium, provided the original work is properly cited. 


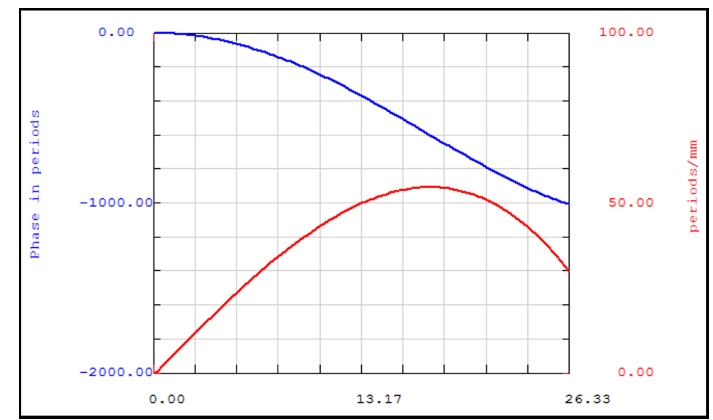

Figure 3. The resulting correction phase function (blue) was transformed to a quasi-periodic transmittance function of the diffractive element (red).

The chromatic aberration of the aspheric lens was analyzed in the ZEMAX software and the correction was computed. The diffractive element was proposed to be directly attached to the back planar surface of the lens (figure 2). The resulting phase function of the correction element is shown in figure 3.

This continuous function can be transformed to a quasiperiodic transmittance function of the diffractive element (also shown in figure 3). Since the refractive lens carries most of the optical power of the system, the highest spatial frequency of the diffractive element can be relatively small and the fabrication requirements remain within the acceptable range. In particular, the spatial frequency is varying over the pupil area from 0 to 55 periods per millimeter (the dependency on the radius is not monotonous). The aberrations of the system with and without the correction are shown in figures 4,5 , and 6 .

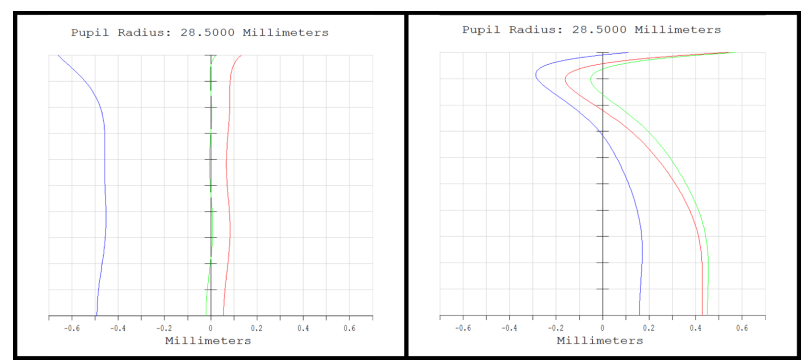

Figure 4. Comparison of the longitudinal aberration of the uncorrected and corrected system.

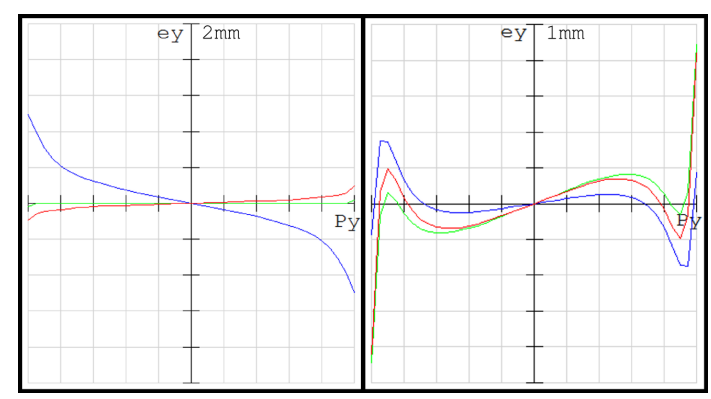

Figure 5. Comparison of the transverse ray fan plot of the uncorrected and corrected system.

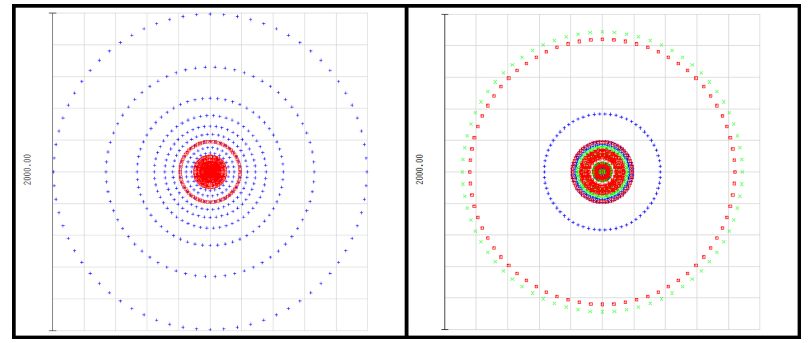

Figure 6. Comparison of the spot diagrams of the uncorrected and corrected system.

\section{Fabrication}

The designed diffractive elements were fabricated using an advanced matrix laser lithography technique [4]. An in-house developed lithograph was used. The technique is based on imaging of the designed micro-structure from a computer driven spatial light modulator onto the surface of the recording material with strong demagnification. The optical setup of the device is displayed in figure 7 .

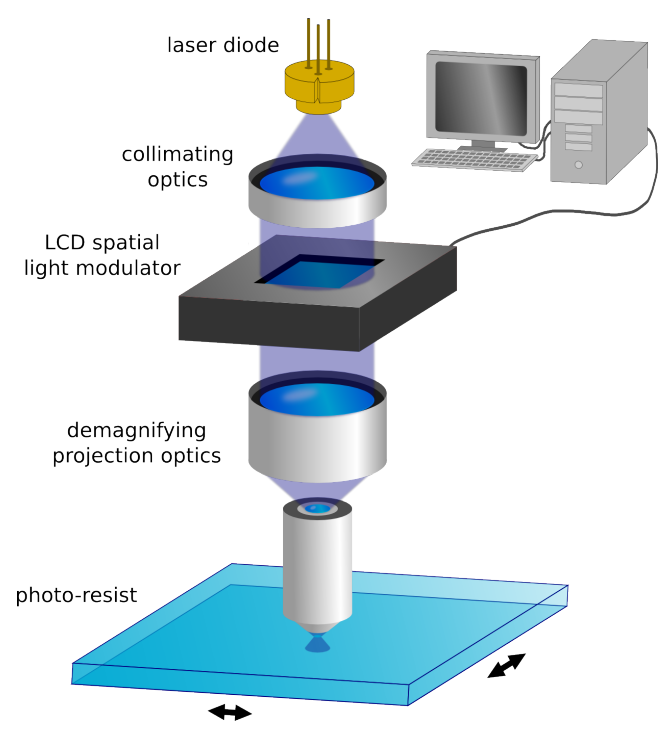

Figure 7. Basic setup of the in-house developed laser writer based on projection of the designed micro-structure from a computer driven microdisplay.

As a light source, the laser diode Nichia NDV4313 is used operating on the wavelength $405 \mathrm{~nm}$. The operating temperature of the diode is stabilized with Thorlabs TCLDM9 cooled diode mount. The output power of the diode is controlled using the Thorlabs ITC110 controller and it is typically $120 \mathrm{~mW}$. The light from the laser is collimated and it illuminates the spatial light modulator Holoeye LC-2002, which is used for projecting the input data (the input data is the blaze profile of the structure converted to a set of grey scale images). The elementary pixel size of the modulator is $32 \mu \mathrm{m}$ and the size of the display is $800 \times 600$ pixels. The image from the modulator is demagnified in an optical system which consists of two objectives. The total demagnification of the system is about $150 \mathrm{x}$, which gives the elementary exposed area of about $130 \times 170 \mu \mathrm{m}$.

The size of the fabricated diffractive elements using the in-house developed lithograph was approximate $50 \mathrm{~mm}$. 
The blazed structure was recorded into the photo-resist material. The exposure and chemical processing was optimized for the particular recording material to achieve the desired shape of the blaze. In the end, the structure was transfered to a polycarbonate using an electro-forming and embossing process. The compatibility with the injection-molding process was then tested by the industrial cooperant. A microphotograph of the central part of the fabricated element is shown in figure 8 .

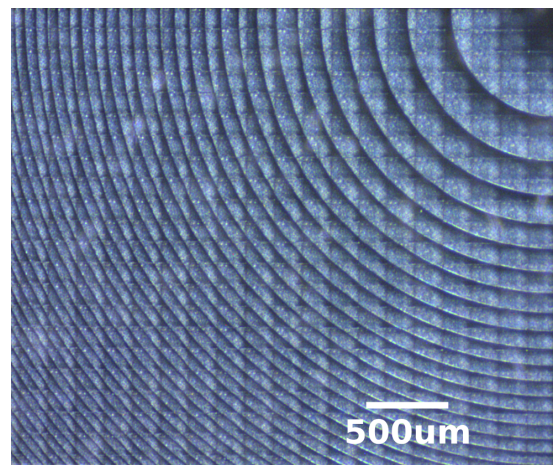

Figure 8. Microscopic image of the central part of the fabricated element.

As it can be seen, the periodicity of the structure changes according to the figure 3 . The blazed shape was measured using an atomic force microscope (AFM) and the image of the final profile can be seen in figure 9 .

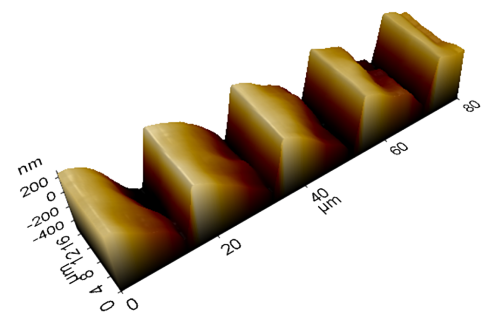

Figure 9. AFM scan of the blazed profile.

\section{Testing}

The fabricated element was tested in the laboratory in a real illuminating system consisting of high power white LED source and the aspheric lens (both provided by the industrial cooperant). The relief diffractive corrector on a planar substrate was directly attached to the lens.

To lower the costs of the prototype, the testing element was fabricated only as one quadrant of the rotationally symmetric structure with radius $28 \mathrm{~mm}$. In figure 10 there is the measurement setup with a mask filtering the three unwanted quadrants of the incident beam. The screen was placed approximately $15 \mathrm{~m}$ from the illumination system. The images on the screen were compared for the setup with and without the diffractive correction. In this setup, the chromatic properties of the beam can be easily measured using an appropriate camera or a spectrometer.

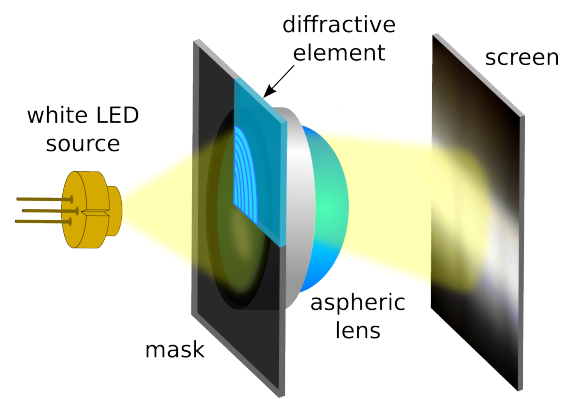

Figure 10. The testing setup with the diffractive correction.

\section{Conclusions}

The diffractive element for the chromatic aberration of illumination system was designed and fabricated. It has been shown, that the correction function can be easily calculated using the ray-tracing methods and can be easily transformed to the transmittance function of a diffractive element. The computed structure was fabricated in photo-resist material using the advanced matrix laser lithography. The exposure and chemical processing was tuned for the particular blazed profile. The correction element was transfered into a nickel substrate and finally to the polycarbonate using the electro-forming and embossing process. The depth of the fabricated structure is approximately $900 \mathrm{~nm}$ (phase shift equivalent to one wavelength for the given material).

In figure 11 there is a comparison of three photographs of the experimental measurements of the system without and with the correction of the chromatic aberration. It is clearly sen that the chromatic aberration of the corrected system is much lower than the aberration of the uncorrected system.

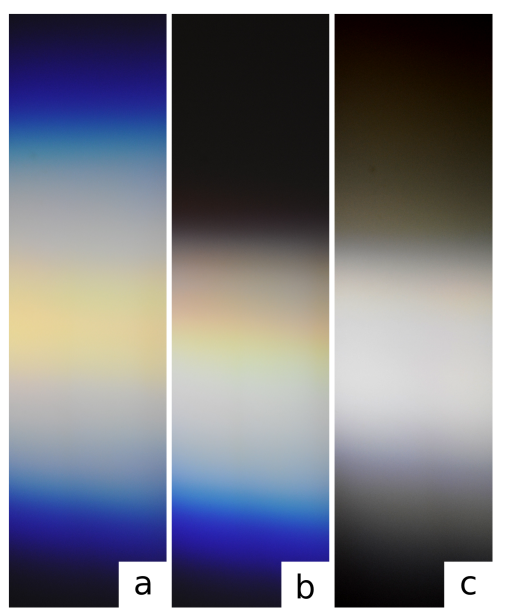

Figure 11. Photos of the illuminated area. (a) full aperture without correction. (b) 1/4 aperture (quadrant mask) without correction. (c) $1 / 4$ aperture with the quadrant mask and the correction element.

Currently, the element is being tested for the robustness and the reliability in the process of fabrication of molded lenses. The blazed structure on a nickel substrate was used as a part of the mold tool by the industrial cooperant. The degradation of the blazed profile on the molded part has been measured using AFM and another corrections for the 
whole manufacturing process are being prepared. The final product is intended to be used in the mass production of the headlights in the automotive industry.

\section{Acknowledgements}

This work was supported by the Ministry of Education of the Czech Republic Research Plan No. 6840770022, by the Grant Agency of the Czech Academy of Sciences Project KAN401220801, and by Visteon-Autopal. Authors would like to thank Dr. David Najdek from Viesteon-Autopal for cooperation and testing of the injection-molding technology for the replication of the fabricated elements.

\section{References}

1. N. Davidson, A. A. Friesem, E. Hasman, Applied Optics, 32, 4770 (1993).

2. N. Bokor, N. Davidson, Applied Optics, 40, 5825 (2001).

3. S. Koth, J. Nowak, M. Zajac, Optik, 106, 63 (1997).

4. M. Škereň, J. Svoboda, M. Nývlt, P. Fiala, Frontiers in Optics (FiO)/Laser Science (LS), FtuM2 (2011). 